REVESCO. Revista de Estudios Cooperativos

ISSN: $1885-8031$

\title{
Un estudio sobre la gestión comunicativa en las cooperativas españolas
}

\author{
Pedro Pablo Marín Dueñas ${ }^{1}$ y y Diego Gómez Carmona ${ }^{2}$ (C) \\ Recibido: 29 de mayo de 2020 / Aceptado: 21 de septiembre de 2021/ / Publicado: 1 de diciembre de 2021
}

Resumen. Partiendo de la premisa de que la actividad comunicativa es un factor clave en el desempeño organizacional este trabajo de investigación analiza la gestión que las cooperativas españolas hacen de su comunicación. Dada la relevancia que estas organizaciones tienen para el desarrollo económico y social del país y la escasez de estudios que abordan este tema, se ha considerado de importancia desarrollar este estudio para determinar si las cooperativas han asumido la comunicación como una herramienta relevante para su desarrollo y si gestionan la misma de una manera adecuada. Para ello se ha aplicado la metodología cuantitativa de la encuesta a una muestra de 338 cooperativas españolas. De los resultados se desprende que las cooperativas, si bien aún no se encuentran en una fase avanzada en cuanto a la gestión de comunicación que realizan si que presentan algunos aspectos en su gestión que permiten ser optimistas de cara al futuro.

Palabras clave: Comunicación empresarial; Planificación; Gestión comunicativa.

Claves Econlit: D83; M30; M31; M38; Q13.

\section{[en] A study on communication management in Spanish cooperatives}

\begin{abstract}
Based on the premise that communication activity is a key factor in organisational performance, this research study analyses how Spanish cooperatives manage their communication. Given the importance of these organisations for the economic and social development of the country and the scarcity of studies on this subject, it was considered important to carry out this study in order to determine whether cooperatives have adopted communication as a relevant tool for their development and whether they manage it appropriately. To this end, the quantitative survey methodology was applied to a sample of 338 Spanish co-operatives. The results show that, although the co-operatives are not yet at an advanced stage in terms of their communication management, they do have some aspects in their management that allow us to be optimistic about the future.
\end{abstract}

Keywords: Business Communication; Planning; Communicative management.

Sumario. 1. Introducción. 2. Objetivos y metodología. 3.Resultados. 4. Conclusiones 5. Referencias bibliográficas.

Cómo citar. Marín Dueñas, P.P.; Gómez Carmona, D. (2021) Un estudio sobre la gestión comunicativa en las cooperativas españolas. REVESCO. Revista de Estudios Cooperativos, vol. 139, e78538. https://dx.doi.org/10.5209/reve.78538.

\section{Introducción}

En el contexto económico actual, marcado por la crisis sanitaria del coronavirus, el desarrollo empresarial es especialmente complicado y son pocas las organizaciones, independientemente del tipo que sean, que no se hayan visto afectadas por las consecuencias económicas que la pandemia ha provocado. Para afrontar esta situación, las compañías de todo tipo, y las cooperativas en particular, por su contribución al progreso económico y social de las comunidades en las que desarrollan su actividad (Muñoz y Tirado, 2014), deben implementar modelos de gestión en sus negocios que les aporten un valor añadido y que les permitan competir en el mercado desde una posición de mayor fuerza.

Y de todas las estrategias posibles, la comunicación que desarrollen para relacionarse e influir en sus públicos se debe configurar como una herramienta clave. En la economía globalizada actual, caracterizada por la denominada sociedad de la información, del conocimiento o sociedad red, la supervivencia y el crecimiento de cualquier empresa debe pasar por el desarrollo de estrategias de comunicación empresarial

\footnotetext{
Universidad de Cádiz, España.

Dirección de correo electrónico: pablo.marin@uca.es.

2 Universidad de Cádiz, España.

Dirección de correo electrónico: diego.gomezcarmona@uca.es.
} 
(Marín, 2016) y las sociedades cooperativas no pueden quedarse al margen. Como apunta Sá (2012), estas organizaciones tienen la necesidad imperiosa de integrar una filosofía de marketing relacional en su gestión.

Cualquier organización, por el hecho de serlo, y sin importar el tipo o el tamaño, comunica, aunque no sean conscientes de ello. En la época actual, la comunicación se convierte en un valor decisivo para las organizaciones. Desde una perspectiva comunicativa, es cierto que a lo largo del siglo XX y, especialmente del siglo XXI, las empresas en general han ido otorgándole mayor protagonismo a la comunicación que desarrollan para relacionarse con sus públicos, experimentando su gestión un crecimiento significativo (Losada, 2009), convirtiéndose en un instrumento generador de valor, diferenciación y liderazgo (Villafañe, 2008). Y es que, como ya señalaban Schmertz y Novak (1986) el silencio no es rentable para ningún tipo de empresa o institución. Las empresas deben mantener una relación personal, fluida y estable con sus públicos y no relegar su comunicación a momentos puntuales o a momentos de crisis.

Si bien esto es cierto, no lo es menos que el desarrollo de estas acciones ha estado asociado, en general, a las grandes empresas o a empresas con grandes recursos, siendo considerada esta actividad como uno de los factores clave para el éxito de los negocios, y adquiriendo una importancia vital en el seno de las mismas. Estas empresas más potentes están girando cada vez más hacia una gestión comunicativa de los intangibles (marcas, patentes, habilidades personales...) que pasan a convertirse en el centro neurálgico de las mismas (Schultz y Kitchen, 2004) y son conscientes de la importancia y de la necesidad de tener planes de comunicación perfectamente estructurados y desarrollados; partidas presupuestarias destinadas a la puesta en marcha de acciones de comunicación; una identidad visual que impacte y cale en la mente del usuario; y estrategias para gestionar su imagen corporativa (Carrillo, Castillo y Tato, 2008).

Cuestión distinta es si otro tipo de empresas con a priori menos recursos, como las cooperativas, han asumido la importancia de la comunicación para su desarrollo y si, además, lo plasman en planes y en una gestión profesional de esta actividad o, por el contrario, esta actividad sigue ocupando un lugar accesorio en sus planteamientos empresariales, siendo asignatura pendiente, aún, el impulso de las estrategias de comunicación.

En un momento tan crítico como el actual, en el que la pandemia del Covid-19 ha provocado una coyuntura económica y social tan negativa, se ha de destacar la importancia que la comunicación tiene para el desarrollo de las empresas, convirtiéndose en uno de los pilares fundamentales para las mismas y constituyéndose en una realidad consustancial al hacer corporativo (Cornelissen, 2011; Sánchez, 2005). Si en un contexto de normalidad, las empresas no pueden seguir ancladas en la producción, ventas y gestión financiera como únicas armas competitivas y dejar de lado la comunicación, dada la actual situación de crisis, si antes era importante para las empresas comunicarse con sus públicos, hoy día se convierte en una necesidad imperiosa y en herramienta clave para su supervivencia. En este sentido, la generación de valor a partir de la comunicación que realizan debe adquirir una relevancia destacada (DIRCOM, 2018), situando a la comunicación empresarial en un lugar estratégico.

En este sentido, se parte de la premisa de que, si bien el estudio de la comunicación en las grandes empresas está más desarrollado, el nivel de análisis en otro tipo de organizaciones, como las cooperativas, se encuentra aún en una fase muy embrionaria, siendo el número de estudios escaso. A pesar de que las estadísticas reflejan la importancia cuantitativa de las cooperativas para el desarrollo de cualquier región por su contribución al progreso económico y social de una comunidad, se observa una carencia de estudios que pongan el foco en el campo de la comunicación empresarial.

Como apuntan Carrillo y Castillo (2007), ante la importancia creciente que la comunicación está tomando para la gestión empresarial, es necesario llevar a cabo investigaciones que analicen el uso de la comunicación por parte de los empresarios, de cara a detectar posibles carencias y errores y ofrecer posibles soluciones. Aspectos como la gestión y el desarrollo de estrategias comunicacionales, conceptos como marca, identidad, imagen o reputación corporativas han sido ampliamente tratados en estudios de diversa índole sobre las grandes organizaciones, multinacionales fundamentalmente, y han demostrado su importancia en las mismas (Marín, Mier-Terán y Lasso de la Vega, 2018). Sin embargo, pocos son los análisis que se han ocupado de analizar cómo las cooperativas desarrollan su comunicación empresarial.

La evolución del marketing y la comunicación constituyen "una oportunidad que la gestión de las organizaciones de la economía social está obligada a considerar, especialmente en estos tiempos que la globalización de mercado liberal mercantil se pretende imponer como modelo único" (Sa, 2012:202). Es por esto que se hace necesario este trabajo de análisis sobre las cooperativas españolas y, más concretamente, sobre la gestión de la actividad comunicativa que desarrollan, por la importancia que tienen para la marcha de las economías locales y por su relevancia social para el entorno en el que desarrollan su actividad. Se aborda un estudio sobre la relevancia que las cooperativas conceden a la comunicación profundizando en cómo planifican la misma, cuánto invierten y quién gestiona esta actividad o qué herramientas implementan de cara a determinar un perfil comunicativo de las cooperativas españolas. 


\subsection{La gestión comunicativa en las organizaciones cooperativas y su importancia}

La comunicación es una herramienta estratégica para el desarrollo de cualquier tipo de organización, convirtiéndose en uno de los pilares fundamentales para las mismas y constituyéndose en una realidad consustancial al hacer corporativo (Cornelissen, 2011; Sánchez, 2005). Sin embargo, son muchas las compañías que tienen poco desarrollada la gestión comunicativa, invirtiendo, además, pocos recursos en esta área. Como señalan Gómez (2011) o Argenty y Barnes (2009), el desarrollo de esta actividad está ligado, en las últimas décadas, especialmente al ámbito de las grandes empresas. En cualquier caso, las organizaciones que desempeñan una actividad económica, como las cooperativas, no pueden seguir ancladas en la producción, ventas y gestión financiera como únicas armas competitivas, dejando de lado la comunicación. Como afirman Herranz de la Casa y Salinas (2004, p. 248):

"No hay que olvidar que las cooperativas son empresas y como tales su funcionamiento depende de la venta de productos y servicios. La comunicación es un instrumento que puede ayudar a esto no sólo en la fase de comercialización sino en todo el proceso. Esta idea es un eje central para que la comunicación adquiera una verdadera importancia estratégica en las cooperativas".

Especialmente en la economía globalizada actual, caracterizada por el desarrollo imparable de Internet y un cambio de valores en la sociedad que demanda nuevas actuaciones por parte del tejido empresarial (Puentes y Velasco, 2009), si antes era importante comunicarse con sus públicos, hoy es una necesidad imperiosa. Y las sociedades cooperativas, consideradas como verdaderas instituciones socioeconómicas, es decir, como auténticas empresas (Bel, 1997), no pueden quedarse al margen.

La comunicación, por tanto, debe convertirse en uno de los pilares fundamentales de la empresa, en un recurso básico para el funcionamiento de toda entidad. El problema es que, en muchas ocasiones, se le concede poca importancia a esta función por parte de las organizaciones. Es habitual que no se dispongan recursos ni económicos ni operativos concretos para resolver problemas relacionados con la comunicación, y que no se observe a ésta como una herramienta de gestión (Carrillo y Tato, 2012). Además, esta comunicación no se plantea desde una perspectiva global e integral, sino que se limita, en muchas ocasiones, a actuaciones de marketing o de publicidad para vender los productos. Muchas empresas desarrollan acciones inconexas y lo hacen de manera fragmentada, lo que se convierte en un obstáculo para controlar y gestionar su comunicación desde un enfoque estratégico, restando, a su vez, eficiencia y coherencia a sus acciones.

Cuando una compañía nace, lo hace con el propósito de desarrollarse y crecer por medio de una actividad económica que le permita generar ingresos y beneficios. Su primera necesidad es contar con una mínima infraestructura con la que pueda fabricar su producto u ofrecer su servicio y en esto centra sus primeras acciones. Secundariamente, se preocupa de difundir su actividad y, además, lo hace con el fin último de conseguir clientes. Este es el planteamiento que muchos gerentes hacen respecto a la comunicación. Toda gira en torno a los recursos y los medios sin dejar cabida a la puesta en marcha de planes de comunicación (Gómez, 2011).

Para Stanton (1989) todas las empresas, con independencia de su tamaño, productos o servicios producidos y situación geográfica, deben comunicarse para sobrevivir. Hoy día, la comunicación se ha convertido en condición indispensable para conseguir una posición favorable en la sociedad y lograr el éxito empresarial. Si bien esta necesidad de comunicar de las organizaciones es tan antigua como la existencia de las mismas, en la actualidad han aparecido nuevas demandas por parte de la sociedad, nuevas audiencias e interlocutores, que a su vez son cada vez más numerosos y están mejor informados, y, por supuesto, un mayor número de nuevos canales aparecidos con el desarrollo de las nuevas tecnologías de la información y de la comunicación que obligan a las empresas a situar a la comunicación como uno de los ejes estratégicos en su gestión. Así mismo, la aparición de la web 2.0 y la hegemonía de las redes sociales "ha cambiado la forma de pensar y actuar de los consumidores" (Pérez y Luque, 2018, p. 257) y ha revolucionado la manera de comunicar de las organizaciones, que deben integrarlas de manera determinante en sus estrategias comunicativas.

La comunicación ya no se puede reducir a la aparición en medios a través de una nota de prensa o la realización de una campaña puntual de publicidad, sino que debe estar vinculada a la estrategia corporativa (Moreno, Molleda, Athaydes, Suárez, Herrera y Álvarez, 2017) y pasar del nivel operativo al nivel de alta gestión permitiendo una adecuada planificación y evaluación de la comunicación (Zerfass y Volk, 2017). No puede limitarse, por tanto, a la suma de un conjunto de acciones aisladas, sino que debe entenderse como un proceso integral, donde se pongan en juego toda una gama de herramientas del campo del periodismo, las relaciones públicas, el marketing, la publicidad, la comunicación interna y, actualmente, la comunicación online y el social media, que cobran especial relevancia hoy día (DIRCOM, 2018) de cara a comunicarse no sólo con su entorno inmediato (clientes, competidores, proveedores y público en general) sino también con otros grupos con los que es importante y necesario establecer una relación. 
En el caso concreto de las cooperativas que participan en los mismos mercados que el resto de las empresas, tienen que ofrecer sus productos y servicios y en consecuencia deben comunicar su existencia de una manera eficaz. El problema es que, tal y como apuntan Herranz de la Casa y Salinas (2004, p. 25):

\footnotetext{
"hay una opinión unánime que apunta que las empresas de la economía social no comunican, no muestran sus proyectos, tienen una falta de iniciativa comunicativa, no tienen cultura de proyectar imagen y desconocen que esto pueda influir en su negocio. En consecuencia, esto provoca que la comunicación, en muchos casos, no exista, sea endogámica (solamente dentro del propio sector de actividad), no sea transparente y sea reducida"
}

Y, en este sentido, las cooperativas, como agentes de desarrollo económico y social de las regiones en las que se integran (Puentes y Velasco, 2009), por su relevancia para el desarrollo rural y como fuente generadora de empleo, presentes en todos los sectores económicos del país, donde a tenor de los datos, su importancia es constatable (Chavez y Monzón, 2008), no pueden obviar la comunicación empresarial puesto que se trata de una herramienta clave para su supervivencia.

Estas organizaciones deben tomar conciencia de la importancia que la comunicación tiene como instrumento estratégico en la gestión de sus negocios. Para lograr sus objetivos, tienen la necesidad de llegar a sus públicos y darse a conocer, no sólo sus productos/servicios, sino también a ellas mismas, como instituciones que crean riqueza para la sociedad.

Como señalan Herranz de la Casa y Salinas (2004, p.33), las empresas de la economía social y las cooperativas en particular "deben empezar a manejar los términos del mundo empresarial actual y superar el miedo a conceptos que manejan las empresas". En definitiva, las cooperativas deben romper con el mito por el cual la comunicación es sólo para las grandes corporaciones y ser conscientes de que quién no se comunica con el medio exterior tiende a su desaparición. En un área donde tienen grandes carencias y lagunas y cuya gestión es deficiente (Salinas, 2004), las cooperativas deben potenciar su comunicación e incorporar en sus estructuras dichas estrategias que les permitan, en el mercado competitivo y complejo en el que desarrollan sus actividades, contar con mayores posibilidades de éxito.

\section{Objetivos y metodología}

A partir de las premisas anteriores se define el objetivo general de esta investigación: analizar la gestión comunicativa que hacen las cooperativas españolas. Este objetivo principal se articula en una serie de objetivos específicos que lo complementan y que servirán de fundamento para el diseño de esta investigación y la elección de las herramientas metodológicas a implementar durante el desarrollo de la misma.

- $\quad$ O.E.1. Medir el grado de importancia que las cooperativas conceden a la comunicación

- $\quad$ O.E.2. Definir los objetivos que las cooperativas quieren alcanzar con su actividad comunicativa

- $\quad$ O.E.3. Determinar si las cooperativas planifican su comunicación

- $\quad$ O.E.4. Conocer quiénes asumen la gestión de la comunicación en las cooperativas

- O.E.5. Determinar cuáles son las principales herramientas de comunicación que las cooperativas implementan en sus procesos comunicativos

- $\quad$ O.E.6. Analizar si las cooperativas evalúan la efectividad de su actividad comunicativa

- O.E.7. Establecer un perfil de las cooperativas en función de la gestión comunicativa que desarrollan

Toda vez que se han establecido los objetivos de la investigación, la encuesta online a partir de un cuestionario estructurado ha sido el instrumento metodológico elegido para lograr la consecución de los mismos ya que permite "obtener, de manera sistemática y ordenada, información de la población investigada sobre las variables o temas que nos interese conocer" (Soler, 2001:21).

Previo a la elaboración del cuestionario, se han definido las siguientes variables de estudio, fundamentadas en los trabajos de Morales (2006), Carrillo y Tato (2008), Blay (2010), Carretón (2010), Pérez (2011), Marín (2016) y a partir de las cuales se ha diseñado el cuestionario.

Concretamente, estas variables son:

1. Perfil de la organización

2. Importancia otorgada a la comunicación

3. Inversión

4. Asunción de tareas de comunicación

5. Objetivos comunicativos

6. Planificación de la comunicación

7. Comunicación digital y de crisis 
8. Herramientas comunicativas

9. Evaluación

10. Perfil comunicativo

Para establecer el perfil comunicativo de las empresas se ha utilizado la propuesta de Carrillo y Tato (2008), que distingue entre:

- Organizaciones básicas primarias: Empresas que no están interesadas en la planificación de cualquier tipo de Comunicación.

- $\quad$ Organizaciones básicas secundarias: Empresas que desarrollan de manera aceptable la planificación de las políticas de Comunicación Comercial o de Marketing pero que muestra menor interés por las políticas de Comunicación Corporativa e Interna y que, sólo puntualmente comunican aspectos relacionados con la propia empresa, de tipo corporativo.

Organizaciones avanzadas: Empresas que desarrollan acciones de Comunicación integradas (comercial, corporativa e interna) y que incluyen la gestión de los activos intangibles en sus estrategias de Comunicación.

De cara a la determinación de la población objeto de estudio y ante la necesidad de contar con un registro de cooperativas de suficiente prestigio y entidad, se recurrió al informe "Las empresas más relevantes de la economía social 2019-2020" que desde 2007 elabora la Confederación Empresarial Española de la Economía Social (CEPES) y que incluye un ranking de cooperativas ordenados por nivel de facturación y que integra en total a 702 entidades cooperativas que se configuran como el universo de estudio. Esto implica que, por tanto, del total de cooperativas españolas realmente este estudio aborda la gestión que hacen las organizaciones que más facturan en España. El cuestionario definitivo se implementó a través de la herramienta Google Forms y ha estado habilitado desde el 30 de abril y hasta el 27 de mayo de 2019. La muestra final, conformada por 338 cooperativas de toda España, se ha seleccionado a través de un muestreo no probabilístico por conveniencia. Se debe aclarar que si bien el cuestionario fue enviado a las 702 cooperativas del listado, el dato de 338 hace referencia a las cooperativas que respondieron al cuestionario, situándose la tasa de respuesta, por tanto, en el $48 \%$. Los resultados han sido analizados con el software estadístico SPSS.

\section{Resultados}

\subsection{Caracterización de la muestra}

La mayoría de cooperativas presentes en la muestra son "maduras" (95\%), es decir, llevan funcionando más de 10 años. Y el $98 \%$ de las analizadas tienen una trayectoria empresarial de más de 5 años, lo que permite afirmar que son empresas consolidadas en el mercado.

Gráfico. 1. Trayectoria de la cooperativa.

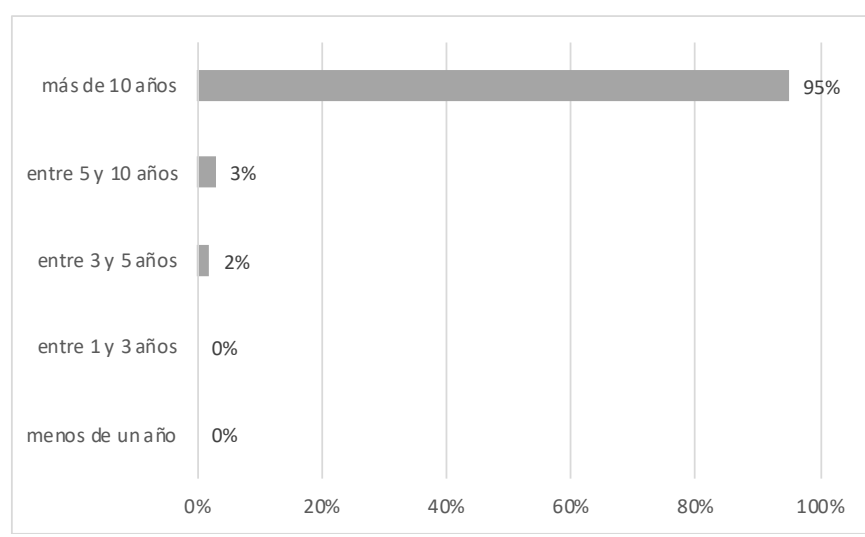

Fuente: Elaboración propia.

En cuanto al tipo de cooperativa, son las agrarias $(63,3 \%)$ el tipo predominante en la muestra, seguida de las cooperativas de trabajo asociado $(15,1 \%)$ y las de servicios $(7,7 \%)$ y ya, en mucho menor medida, las de consumidores y usuarios $(5,3 \%)$, de iniciativa social $(4,4 \%)$ o de enseñanza $(2,7 \%)$. 
Gráfico. 2. Tipo de cooperativa.

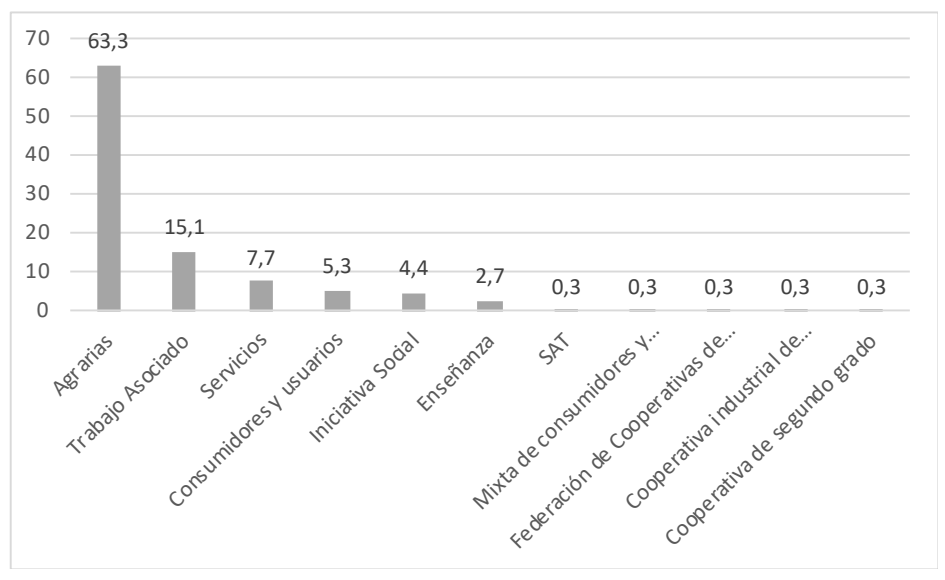

Fuente: elaboración propia

El tamaño empresarial es otra de las características más estudiadas en las investigaciones científicas que tratan de determinar el comportamiento empresarial (Arteaga y Medina, 2006) por lo que resulta interesante determinar si existen diferencias entre la dimensión de las mismas en cuanto a la gestión comunicativa que realizan.

En función del número de empleados, en la muestra predominan las pequeñas y medianas empresas (de 10 a 249 empleados-51,7\% de la muestra). Por su parte, las empresas unipersonales y las microempresas, que caracterizan el tejido empresarial español, sólo suponen el 21,6\% de la muestra, mientras que las cooperativas que se consideran grandes empresas (250 empleados o más), estás suponen el 26,7\%.

Gráfico. 3. Tamaño de las cooperativas.

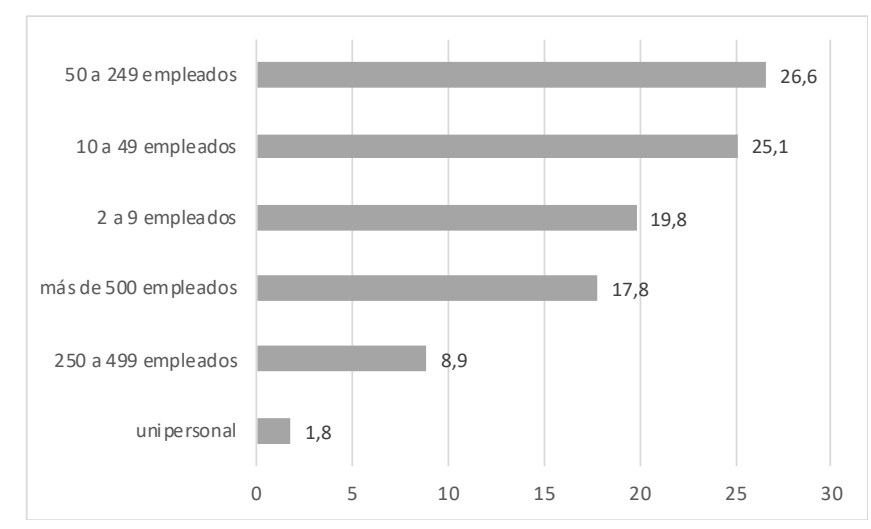

Fuente: elaboración propia

En cuanto al ámbito territorial en el que desarrollan su actividad, el 50\% de la muestra lo hace a nivel internacional, mientras que un $23,1 \%$ la realiza a nivel nacional y un 16,5 en el ámbito autonómico. Tan sólo el $10,7 \%$ desarrolla su actividad exclusivamente a nivel local. 
Gráfico. 4. Ámbito territorial en el que desarrollan su actividad.

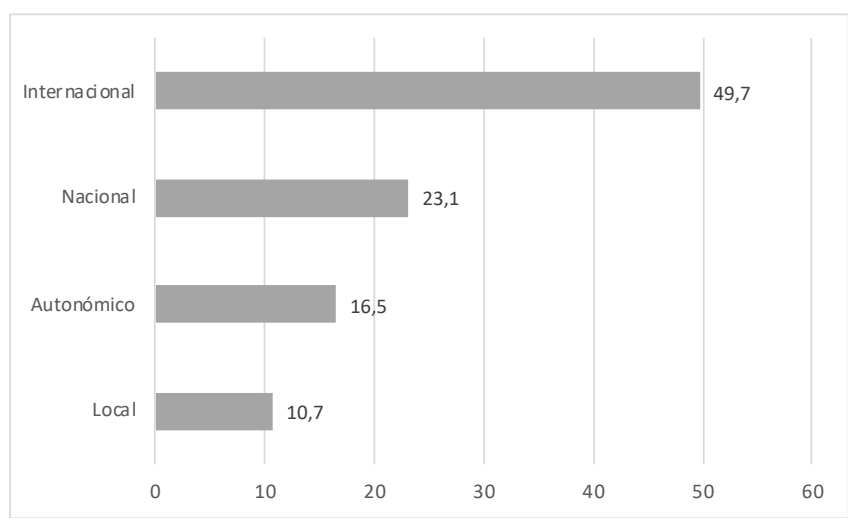

Fuente: elaboración propia

\subsection{Análisis de la gestión comunicacional}

El primer aspecto sobre el que se les ha cuestionado a las cooperativas, a través de una escala Likert 1-5 (siendo 1 nada importante y 5 fundamental) es la importancia que le conceden a la comunicación, dentro de la gestión empresarial. Estas empresas le dan mucha importancia a la comunicación en su estrategia empresarial y la consideran como fundamental o muy importante (87,6\%). La media, se sitúa en el 4,3, para una desviación de ,730. De hecho, ninguna la considera como nada importante y tan sólo el 1,8\% considera que dicha comunicación es algo importante.

Gráfico. 5. Importancia de la comunicación para las cooperativas.

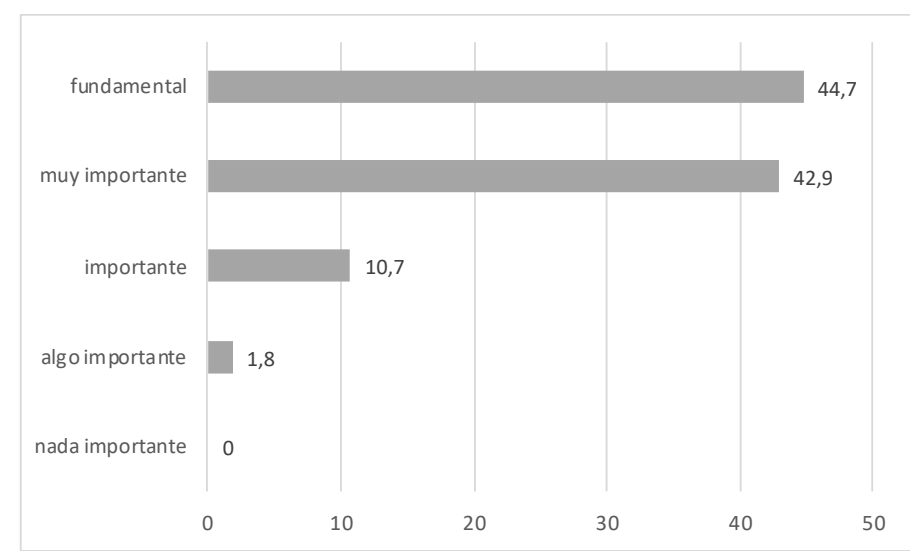

Fuente: elaboración propia

Cuestión distinta es cuando se pregunta por un dato más tangible como la inversión que hacen en comunicación. Los datos que se desprenden de las respuestas a la pregunta sobre la inversión en comunicación no pueden entenderse de manera positiva. El porcentaje de organizaciones que invierten menos del 5\% de su presupuesto en el desarrollo de la comunicación es del 81,9\%, por tan sólo un 18,1\% que invierte entre el $5 \%$ y el $20 \%$. También es de destacar que ninguna cooperativa invierte más del $20 \%$ en comunicación.

Si bien estas organizaciones conceden un alto grado de importancia a la comunicación, esto no se ve reflejado en la inversión que destinan a la misma. Una correcta gestión comunicacional requiere de una inversión adecuada puesto que sin los fondos necesarios no se podrán llevar a cabo actuaciones idóneas. Interesante será profundizar en futuras investigaciones sobre los motivos por los que esta inversión es tan baja. 
Gráfico. 6. Inversión en comunicación

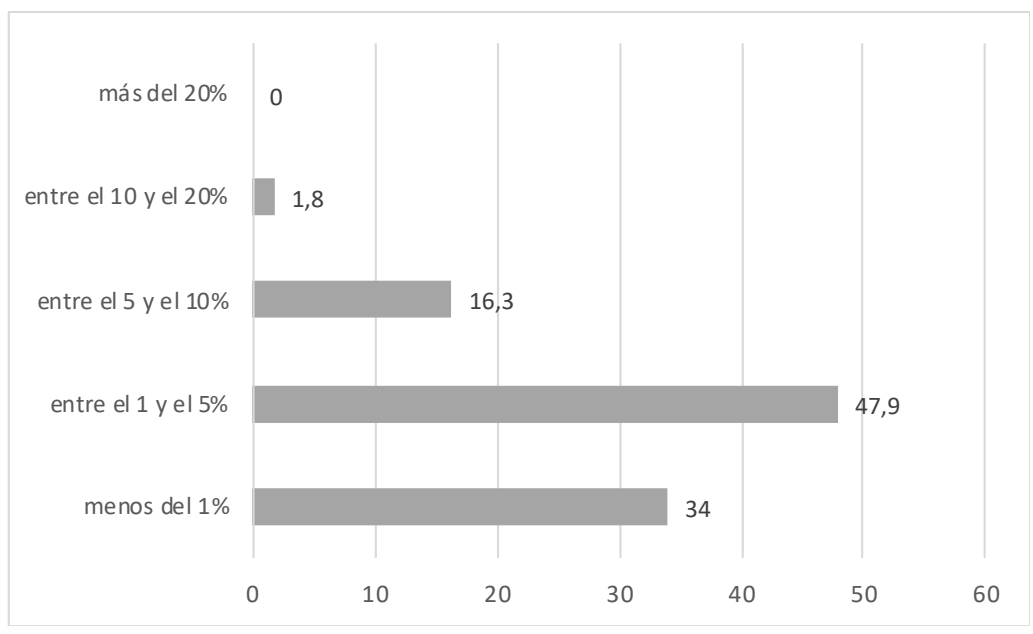

Fuente: elaboración propia

El análisis de la asunción de las tareas de comunicación por parte de las empresas, si bien no es del todo negativo, tampoco invita al optimismo. Por un lado, en tan sólo el 7,1\% nadie asume las tareas de comunicación, lo que significa que en el 93\% restante al menos hay una persona responsable de gestionar la comunicación y, por tanto, las cooperativas son conscientes de que hay que dedicar recursos humanos a esta tarea. Pero, en contraposición, si se profundiza en ese dato, el porcentaje es engañoso porque en el 54,4\%, esta persona desempeña más funciones dentro de la empresa que, si bien no se especifican, implican que ese trabajador no se está dedicando únicamente a gestionar la comunicación de la cooperativa. Por tanto, realmente en tan sólo el 37,3\% de los casos el desempeño de estas funciones se realiza en exclusiva, ya sea por un departamento $(28,4 \%)$ o una persona $(8,9 \%)$.

Este dato también sigue la línea de lo analizado anteriormente. Es decir, las cooperativas le conceden una alta importancia a la gestión comunicativa pero no invierten en ella y además tampoco cuentan con personal en exclusiva para encargarse de la misma. En más del 50\% de los casos analizados una persona se dedica a varias funciones dentro de la organización, entre ellas la comunicación, por lo que difícilmente podrá hacer una correcta gestión de la misma, al no encargarse en exclusiva de una tarea que necesita de una dedicación exclusiva para poder funcionar de manera correcta. Derivado de este resultado se abre una línea de investigación futura que permita, por un lado, identificar los perfiles formativos de las personas que gestionan la comunicación en las cooperativas y, por otro, qué otras tareas realizan aquellos encargados de desempeñar las labores de comunicación.

Gráfico. 7. Asunción de las tareas de comunicación.

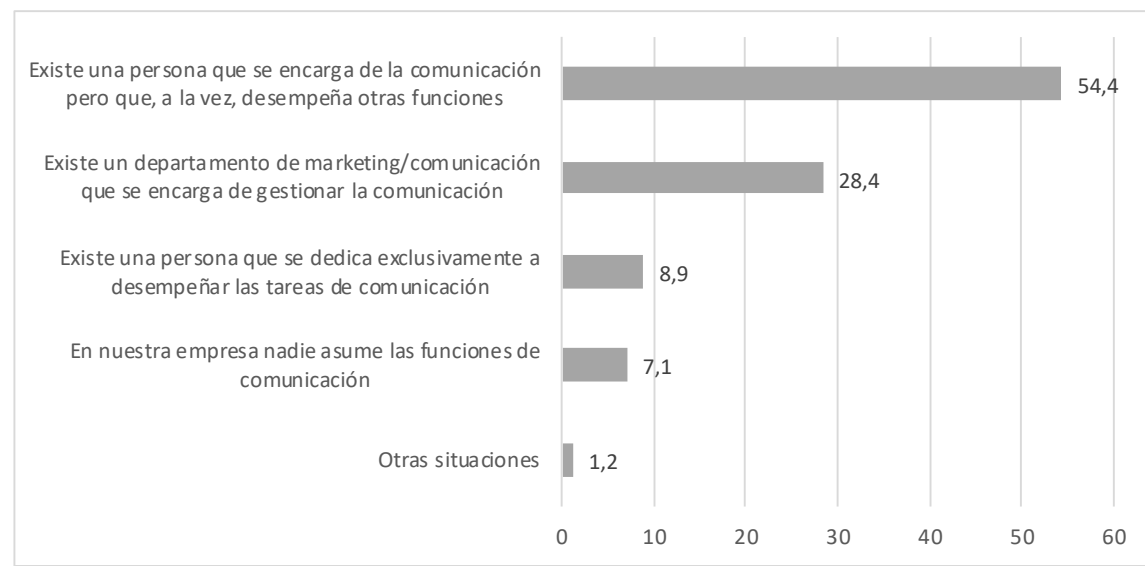

Fuente: elaboración propia

Cuestionadas las empresas sobre los objetivos que buscan con la puesta en práctica de sus acciones, éstos son diversos, si bien destacan aquellos vinculados con la imagen (incrementar la notoriedad de la marca (62\%) o mejorar la imagen de la empresa (71\%)). También es de destacar la relevancia de los objetivos vinculados con la comunicación interna (69\%), si bien es algo lógico dadas las características 
organizacionales propias de estas cooperativas. Aunque en menor medida, también están presentes en su planificación los objetivos vinculados con aspectos de tipo comercial y de ventas, como pueden ser aumentar los nuevos clientes $(48,5 \%)$, vender productos/servicios $(59,2 \%)$ o fidelizar a los consumidores $(60,7 \%)$.

Estos resultados son muy relevantes ya que se comprueba como las cooperativas, a la hora de definir sus objetivos, están en línea con la filosofía empresarial actual que pone el foco en la gestión de la imagen y el desarrollo del branding para llegar a sus públicos anteponiéndolo, incluso, a la comunicación más centrada en la comercialización de los productos.

Gráfico. 8. Objetivos de la comunicación.

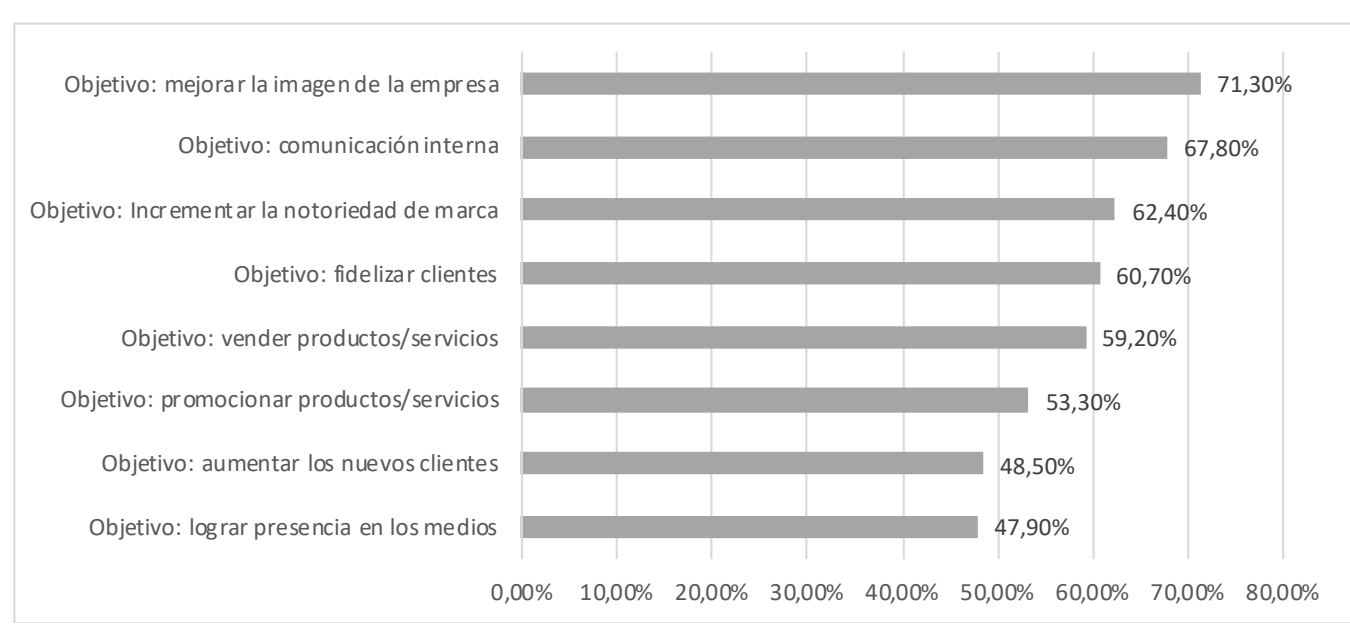

Fuente: elaboración propia

Otro aspecto importante en la gestión comunicacional es la existencia de planes de comunicación que guíen las decisiones y establezcan un marco sobre el que desarrollar las estrategias y acciones comunicativas. En este caso, un 51,5\% admite contar con un plan de comunicación por un 48,5\% que no lo hace, por lo que tan sólo en la mitad de las cooperativas analizadas las decisiones no se toman de forma intuitiva e improvisada. El problema es que estos datos empeoran cuando se estudia la gestión de las acciones de comunicación digital y la comunicación de crisis. En el $61 \%$ de los casos no existe un plan de marketing digital y, desde el punto de vista de la planificación de la comunicación de crisis, el dato empeora aún más. El $70 \%$ reconoce no contar con una planificación estratégica que permita afrontar una crisis en mejores condiciones.

Tal y como se desprende de los resultados previos, estos datos son lógicos ya que las cooperativas no invierten en comunicación ni cuentan, en la mayoría de los casos, con personal en exclusiva dedicado a su gestión, por lo que difícilmente podrán desarrollar planes que guíen la dicha actividad.

Tabla. 1. Planificación de la comunicación.

\begin{tabular}{|l|c|c|}
\hline & SÍ & NO \\
\hline $\begin{array}{l}\text { Existencia de planes de } \\
\text { comunicación }\end{array}$ & $51,5 \%$ & $48,5 \%$ \\
\hline $\begin{array}{l}\text { Existencia de planes de } \\
\text { marketing digital }\end{array}$ & $39,3 \%$ & $60,7 \%$ \\
\hline $\begin{array}{l}\text { Existencia de planes de } \\
\text { comunicación de crisis }\end{array}$ & $30,2 \%$ & $69,8 \%$ \\
\hline
\end{tabular}

Fuente: elaboración propia

Con respecto a las herramientas de comunicación implementadas, destaca el desarrollo de acciones vinculadas a la comunicación digital tales como social media marketing, e-mail marketing, publicidad pagada en internet y contar con una web como canal de comunicación propio (casi el $93 \%$ de las cooperativas cuenta con su propia web) y que confirman el peso cada vez mayor que los canales online tiene 
en la comunicación de las empresas hoy día (Túñez, Costa y Míguez, 2017). Esto significa que estas organizaciones también están en línea con las tendencias actuales en lo que a comunicación se refiere, pues centran su actividad comunicacional en el entorno digital y en el denominado social media marketing. Otras acciones más tradicionales que también destacan entre las más implementadas por las cooperativas son la asistencia a ferias y exposiciones, los patrocinios, la publicidad en prensa, o el desarrollo de catálogos, folletos o similares.

Por el contrario, entre las herramientas que menos desarrollan se pueden encontrar, entre otras: publicidad en televisión o cine, el mailing (buzoneo), la venta personal, el merchandising o herramientas específicas de comunicación interna.

Gráfico. 9. Herramientas de comunicación utilizadas.

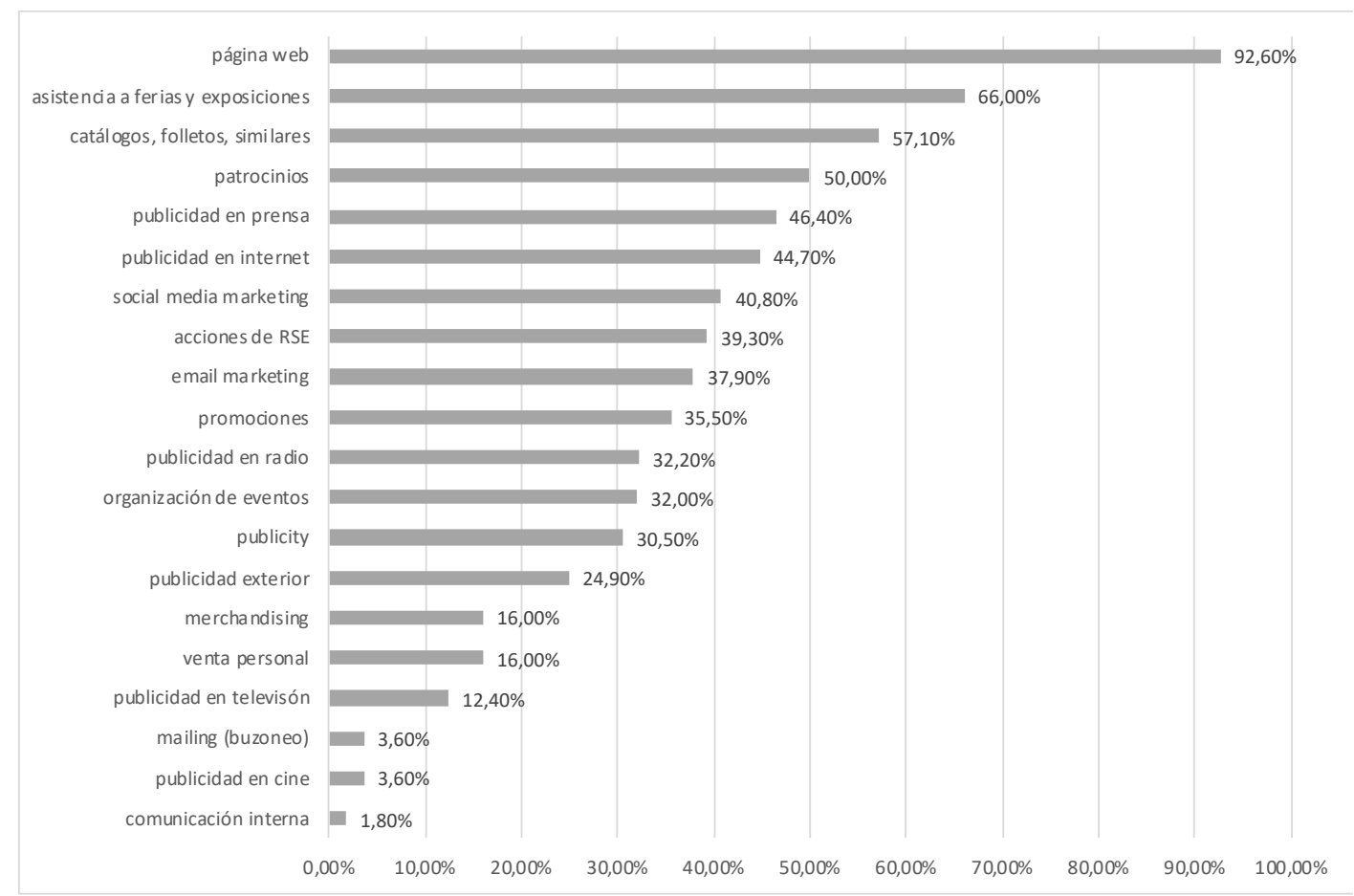

Fuente: elaboración propia

El último aspecto que se ha medido y que también es indicativo de una buena gestión comunicativa por parte de las compañías es el de evaluar las estrategias y acciones que se ponen en práctica e, igualmente, los resultados no son todo lo positivos que cabría esperar, si bien es cierto que en la actualidad las organizaciones apenas evalúan su desempeño en comunicación (Castillo y Villabona, 2018). En el caso de las cooperativas, un $50 \%$ de los encuestados admite poner en práctica acciones para medir la eficacia de su comunicación.

Gráfico. 10. Evaluación de la comunicación.

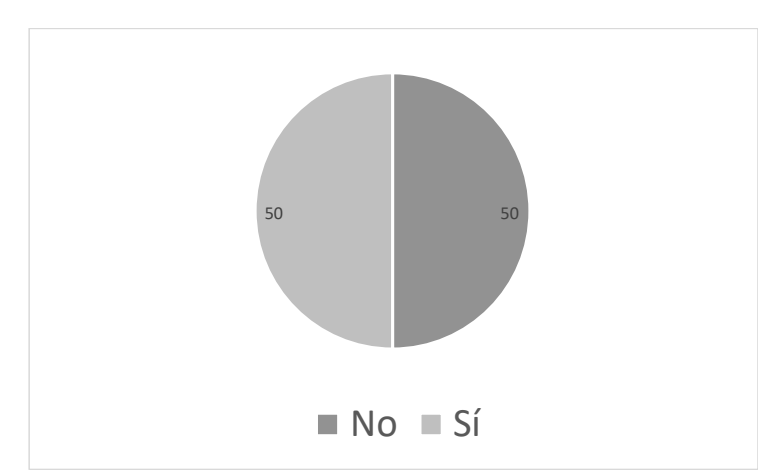

Fuente: elaboración propia 
Como se desprende de estos resultados, se puede afirmar que las cooperativas no sólo son conscientes de la importancia de la comunicación para el desarrollo empresarial, sino que están al día en cuanto a las tendencias comunicativas actuales que ponen el foco en el branding, la imagen corporativa y el social media marketing. El problema es que la realidad de la gestión no ayuda a esta visión. Las cooperativas no invierten en comunicación y esto tiene su reflejo en la gestión comunicacional: no cuentan con personal en exclusiva y tampoco planifican. Esto, como se ha afirmado previamente, abre nuevas líneas de investigación que se centren en analizar los motivos por los que esta inversión es tan baja y los perfiles de las personas que gestionan dicha comunicación. Igualmente, también sería interesante ampliar la muestra y establecer comparativas entre los distintos tipos de cooperativas existentes para analizar las diferencias que puedan darse.

\section{Conclusiones}

Esta investigación partía de la premisa de que la comunicación debe ser una herramienta estratégica para las empresas, independientemente del tipo que sean, siendo esta afirmación especialmente aplicable en situaciones de crisis. En este sentido, las medidas derivadas del estado de alarma decretado por el gobierno español han colocado a muchas organizaciones entre las que, como no puede ser de otra manera, se encuentran las cooperativas, en una situación de gran incertidumbre. Si para cualquier compañía la comunicación con sus públicos es importante, en la actualidad y en los meses que están por venir, esta actividad debe cobrar una relevancia determinante para su supervivencia.

Esta investigación ha medido la gestión que de la comunicación hacen 338 cooperativas españolas con el fin de determinar cómo gestionan su comunicación empresarial, considerando que aquellas que implementen una adecuada gestión comunicativa, tendrán cierta ventaja y partirán de una mejor posición a la hora de pelear en el mercado. Y, para ello, se han medido una serie de variables clave tales como la importancia dada a la comunicación, el porcentaje de inversión, la existencia de planes estratégicos y de contingencia y los tipos de objetivos que se persiguen, así como las acciones que implementan o la aplicación de medidas de medición de resultados, entre otras. Con este análisis se quería, así mismo, establecer un perfil de las cooperativas en función de cómo gestionan su comunicación, partiendo de la propuesta de Carrillo y Tato (2008), que distinguen entre organizaciones básicas primarias, organizaciones básicas secundarias y organizaciones avanzadas.

Los resultados del estudio de estos indicadores permiten afirmar que las cooperativas analizadas se colocan en un nivel intermedio entre organizaciones avanzadas y un nivel básico secundario de gestión comunicativa: es evidente que, en general, estas compañías están interesadas en la comunicación, pero su gestión desde una perspectiva estratégica y planificada no es mayoritaria. Igualmente, si bien cuentan en sus estructuras con capital humano dedicado a esta actividad, el número de cooperativas que cuentan con departamentos o personal en exclusiva desempeñando esta función no es alto. Por otro lado, destinan pocos recursos económicos a la comunicación, y la inversión en la actividad comunicativa debería crecer.

Desde un punto de vista positivo hay que entender que los objetivos comunicativos se plantean en términos corporativos más que comerciales y mejorar la imagen, alcanzar notoriedad, fidelizar clientes o fomentar la comunicación interna destacan entre los fines de estas empresas, por encima de otros más marketinianos como promocionar y vender productos y servicios. Además, muchas de las acciones que realizan se implementan en el entorno digital, por lo que están en línea con la corriente actual que pone el foco de la actividad comunicativa en el medio Internet (Rodríguez y Vázquez, 2019), centrándose en acciones de social media (Stelzner, 2019).

Cabrera y Almansa (2016) consideran que el éxito organizativo pasa por contar con una comunicación estratégica. En el caso de las cooperativas analizadas, por tanto, la importancia dada a la comunicación como función estratégica de gestión, la existencia de profesionales en sus organigramas, así como la inversión en esta actividad evidencian un interés medio por las políticas de comunicación, por lo que deberían seguir avanzando en esta gestión. Si bien el punto de partida no es malo, tienen que seguir potenciando esta actividad, dando un impulso a su comunicación a través de la inversión y la mayor planificación.

En definitiva, incorporar aún más, en su gestión diaria, el desarrollo de las estrategias comunicacionales y la planificación, lo que permitirá a las cooperativas competir en los mercados desde una mejor posición lo que puede favorecer su éxito, entendiendo por éxito afrontar la crisis económica que se avecina en unas mejores condiciones que permitan su supervivencia y su continuidad y, porque no, su crecimiento. El futuro de las cooperativas, enfrentadas a una gran competencia y a un entorno muy cambiante, pasa en gran medida por su capacidad de gestión estratégica en general y, más específicamente, por su capacidad para gestionar su comunicación. 


\section{Referencias bibliográficas}

Argenti, P. A., y Barnes, C. M. (2009) Digital strategies for powerful corporate communica- tions. New York, NY: McGraw-Hill.

Arteaga Ortiz, J y Medina Múñoz, D. R. (2006) La importancia del tamaño en la actividad exportadora: una evaluación en el caso de las PYMES españolas exportadoras no consolidadas. Información Comercial Española, $\mathrm{N}^{\circ}$ 2883, pp. 41-54.

Bel Durán, P. (1997) Las cooperativas agrarias en España. Análisis de los flujos financieros y de la concentración empresarial. Valencia: CIRIEC.

Blay Árraez, R. (2010) Gestión y estrategias de comunicación corporativa en los sectores tradicionales de la comunidad valenciana. Claves para la integración y desarrollo de políticas de comunicación como activo estratégico empresarial. Tesis doctoral. Universitat Jaume I. https://www.tdx.cat/handle/10803/48704.

Cabrera Cabrera, Ma. A. y Almansa Martínez, A. (2016) El director de relaciones públicas en las grandes empresas españolas. Revista Internacional de Relaciones Públicas, vol. $6, \quad \mathrm{~N}^{\circ} \quad 11, \quad$ pp. 113-134. http://dx.doi.org/10.5783/RIRP-11-2016-07-113-134.

Carretón Ballester, M ${ }^{\text {a }}$ C. (2010) Actitud y actividad de las empresas alicantinas en la comunicación con sus públicos. En Carretón-Ballester, $\mathrm{M}^{\mathrm{a}}$ Carmen y Ordeix-Rigo, Enric (Eds.). Las relaciones públicas en la sociedad del conocimiento. Alicante: Asociación de Investigadores en Relaciones Públicas (AIRP), pp. 86-105.

Carrillo Durán, V.; Castillo Díaz, A. y Tato Jiménez, J. L. (2008) El valor de lo intangible. La gestión de la reputación corporativa. El caso de la marca Telefónica. Observatorio (OBS*) Journal, 7, pp. 239-254.

Carrillo Durán, Ma . V. y Tato Jiménez, J. L. (2008) La comunicación empresarial del siglo XXI. La gestión de los activos intangibles. Portal de la Comunicación.

Carrillo Durán, M. V. y Tato Jiménez, J. L. (2012) La Comunicación Empresarial del Siglo XXI. La Gestión de Activos Intangibles. Portal Comunicación. Com.

Castillo Esparcia, A. y Villabona Beltrán, D. (2018) Evaluación para demostrar la efectividad en comunicación y relaciones públicas. Una mirada a las prácticas profesionales desde los Communication Monitor. Communication papers, vol. 7, $\mathrm{N}^{\mathrm{o}} 15$, pp. 137-153.

Confederación Empresarial Española de la Economía Social (CEPES) (2020) Las empresas más relevantes de la economía social 2019-2020. Disponible en: https://www.cepes.es/noticias/620 informe-cepes-las-empresas-masrelevantes-economia-social-2019-2020-muestra-fortaleza-liderazgo-este.

Cornelissen, J. (2011) Corporate communication: A guide to theory and practice. Sage.

Chaves, R. Monzón, J.L. (2008) La economía social en la Unión Europea. CIRIEC.

DIRCOM (2018) El estado de la comunicación en España. https://envios.dircom.org/docs/ECE-2018.pdf.

Gómez Nieto, B. (2011) La comunicación como instrumento de expansión en la pequeña y mediana empresa española: el caso segoviano. Correspondencias \& Análisis, No 1, pp. 157-172.

Herranz de la Casa, J. M. y Salinas, F. (2004) La comunicación en el ámbito de la economía social. Servicio de publicaciones de la Universidad de Ávila.

Losada Díaz, J. C. (2009) Los 10 frentes de la comunicación corporativa. Doxa.comunicacion, No 9, pp. 145-164.

Marín Dueñas, P.P. (2016) La comunicación empresarial en regiones con un bajo desarrollo empresarial: el caso de las PYMES del sur de Europa. Observatorio (OBS*) Journal, vol. 10, $\mathrm{N}^{\circ}$ 1, pp. 151-180.

Marín Dueñas, P. P.; Mier-Terán Franco, J. J. Y Lasso de la Vega González, M. C. (2018) La gestión de la comunicación en la empresa familiar. Cuadernos de Estudios Empresariales, $\mathrm{N}^{\mathrm{o}}$ 28, pp. 55-78.

Morales Serrano, F. (2006) La Comunicación Planificada: estudio cualitativo de las variables estructura, gestión y valores en la comunicación de las organizaciones. Tesis Doctoral. Universidad Autónoma de Barcelona.

Moreno, Á.; Molleda, J.C.; Athaydes, A.; Suárez, A. M.; Herrera, M. y Álvarez, A. (2017) Latin American Communication Monitor 2016 - 2017. Tendencias en comunicación estratégica: big data, automatization, engagement, influencers, coaching y competencias. Resultados de una encuesta en 17 países. https://bit.ly/3pgxZWH.

Muñoz Posadas, R. y Tirado Valencia, P. (2014) Responsabilidad social y transparencia a través de la web: un análisis aplicado a las cooperativas agroalimentarias españolas. REVESCO. Revista de Estudios Cooperativos, Primer cuatrimestre, $\mathrm{N}^{\circ} 114$, pp. 84-105.

Pérez Curiel, C. y Luque Ortiz, S. (2018) El marketing de influencia en moda. Estudio del nuevo modelo de consumo en Instagram de los millennials universitarios. AdComunica. Revista de Estrategias, Tendencias e Innovación en Comunicación, 15.

Pérez Seoane, J. (2011) Estrategias de comunicación textil en la eurorrexión Galicia-Norte de Portugal. Razón y Palabra, 74.

Puentes Poyatos, R. y Velasco Gámez, Má. M. (2009) Importancia de las sociedades cooperativas como medio para contribuir al desarrollo económico, social y medioambiental, de forma sostenible y responsable. REVESCO. Revista de Estudios Cooperativos, Tercer Cuatrimestre, $\mathrm{N}^{\circ}$ 99, pp. 104-129.

Rodríguez Fernández, L. y Vázquez Sande, P. (2019) Retos y perspectivas en la comunicación organizacional. El profesional de la información, vol. $28, \mathrm{~N}^{\circ} 5$.

Sá, J. de (2012) Las prácticas de gestión de marketing en las cooperativas portuguesas. CIRIEC-España, Revista de Economía Pública, Social y Cooperativa, № 76, pp. 199-227. 
Sánchez Calero, M.L. (2005) Desarrollo de la comunicación externa en la empresa. Sala de Prensa. Web para profesionales de la comunicación Iberoamericanos, $\mathrm{N}^{\circ} 83$.

Schmertz, H. y Novak, W. (1986) El silencio no es rentable: El empresario frente a los medios de comunicación. Barcelona: Planeta.

Schultz D. E. y Kitchen Ph. J. (2004) Managing the Changes in Corporate Branding and Communication: Closing and Re-opening the Corporate Umbrella. Corporate Reputation Review, 6 (4), pp. 347-366.

Soler Pujals, P. (2001) Investigación de mercados. Barcelona: Servicio de publicaciones de la Universidad Autónoma de Barcelona.

Stelzner, M. (2019) Social Media Marketing Industry Report. San Diego: SocialMedia

Stanton, N. (1989) Las técnicas de comunicación en la empresa. Bilbao: Ediciones Deusto.

Túñez López, M.; Costa Sánchez, C. y Míguez González, M. I. (2018) Avances y retos de la gestión de la comunicación en el siglo XXI. Procesos, necesidades y carencias en el ámbito institucional. Estudios sobre el Mensaje Periodístico, vol.24, No 1, pp. 921-940.

Villafañe, J. (2008) La comunicación empresarial y la gestión de los intangibles en España y Latinoamérica. Madrid: Pearson Educación.

Zerfass, A., y Volk, S. (2017) Beyond creativity: How communication departments contribute to corporate success. Paper presented at the Annual Congress of the European Public Relations Education and Research Association (EUPRERA), London, UK. 